\title{
An evaluation of eye movement desensitization and reprocessing therapy delivered remotely during the Covid-19 pandemic
}

\author{
lain W. McGowan ${ }^{1 *}$, Naomi Fisher ${ }^{2}$, Justin Havens ${ }^{3}$ and Simon Proudlock ${ }^{4}$
}

\begin{abstract}
Background: In addition to having a negative impact on the physical and emotional health of the population, the global Covid-19 pandemic has necessitated psychotherapists moving their practice to online environments. This service evaluation examines the efficacy of Eye Movement Desensitization and Reprocessing (EMDR) Therapy delivered via the internet.

Methods: A real-world service evaluation was conducted from a self-selecting group of EMDR therapists that subscribe to either a JISCMail discussion list or either the UK or All Ireland National EMDR Associations. Author designed questionnaires were used to gather information on the efficacy of EMDR delivered online as well as client and therapist characteristics.

Results: Thirty-three therapists provided efficacy data on a total of 93 patients. Statistically significant and clinically meaningful reductions were found in all four-psychometrics used both in adult and children and young people populations. Client outcome was not related to therapist experience.
\end{abstract}

Conclusions: EMDR delivered via the internet can be an effective treatment for clients experiencing mental health issues.

\section{Background}

Eye Movement Desensitization and Reprocessing therapy (EMDR) is an effective, evidence-based treatment for the treatment of several mental health issues including posttraumatic stress disorder (PTSD), depression, anxiety and eating disorders. It is recommended as a first line intervention for people experiencing symptoms associated with PTSD by the ISTSS [1] and the World Health Organization [2]. The UK, National Institute of Health and Care Excellence (NICE) [3] recommend using EMDR where trauma focused Cognitive Behavioural Therapy (CBT) is unsuccessful or the client declines CBT.

\footnotetext{
* Correspondence: i.mcgowan@qub.ac.uk

${ }^{1}$ School of Nursing and Midwifery, Queens University, Belfast, UK

Full list of author information is available at the end of the article
}

EMDR is guided by the adaptive information processing model [4] The model posits that current PTSD symptomatology is a result of maladaptive information processing of unprocessed memories stored in the brain. EMDR therapy suggests that after a traumatic experience, information processing is developed using bilateral stimulation of each brain hemisphere "resulting in new learning, elimination of emotional distress, and development of cognitive insights" [5].

The Covid-19 pandemic has had a negative impact on the mental health of the general population. In the last year, several meta-analyses have been published reporting the negative impact Covid-19 has had on the general public [6], healthcare workers [6], people living with physical health problems such as cancer [7] and those with pre-existing mental health issues [8]. This impact

C The Author(s). 2021 Open Access This article is licensed under a Creative Commons Attribution 4.0 International License, which permits use, sharing, adaptation, distribution and reproduction in any medium or format, as long as you give appropriate credit to the original author(s) and the source, provide a link to the Creative Commons licence, and indicate if changes were made. The images or other third party material in this article are included in the article's Creative Commons licence, unless indicated otherwise in a credit line to the material. If material is not included in the article's Creative Commons licence and your intended use is not permitted by statutory regulation or exceeds the permitted use, you will need to obtain permission directly from the copyright holder. To view a copy of this licence, visit http://creativecommons.org/licenses/by/4.0/ The Creative Commons Public Domain Dedication waiver (http://creativecommons.org/publicdomain/zero/1.0/) applies to the data made available in this article, unless otherwise stated in a credit line to the data. 
has been felt globally. In China, Hao et al. (2020) [8] surveyed 76 people currently in treatment for a mental health problem and 109 without. They found statistically significant differences between the group in levels of PTSD symptoms experienced, depression, anxiety and insomnia. Similar findings are reported in Saudi Arabia [9], Pakistan [10] and the Philippines [11]. In the United Kingdom, Pierce et al. [12] report a significant decline of the mental health of the general population between the start of the pandemic and April 2020. Negative impacts of Covid 19 are found in the general population in Spain [13], amongst the student population in Greece [14] and the Philippines [11], and amongst healthcare workers in Italy [6].

Most Government responses to the Covid 19 pandemic included some form of, what became colloquially known in the UK as 'lockdown'. However, social isolation and withdrawal have long been associated with the onset of mental health problems [15]. Consequently, the potential for harm from protective policies is apparent. Tien- Huy et al. (2021) [16] conducted a global survey across 63 countries to assess the impact of quarantine/ isolation on the psychological well-being of individuals $(n=1871)$. They found a statistically significant positive association between the number of days in quarantine and increased perceived stress levels. A significant positive association was also found between perceived stress levels and being exposed to a suspected or a confirmed positive case of Covid-19 [16].

Similar findings are reported by the TMGH-Global COVID-19 Collaborative (2021) [17]. This collaborative sought to examine the presence of PTSD in individuals in insolation/ quarantine. Drawing on results from 944 responses across 57 countries, they report that people experiencing symptoms of Covid - 19 and were forced to isolate reported more PTSD than those isolating for other reasons. Being made to isolate also increased the risk of developing PTSD in comparison to voluntarily entering a period of quarantine (OR: 2.92: 95\% CI: $1.84-$ 4.74: $p<0.001$ ).

It is important to recognise that, in addition to the pandemic, governments and their populations have also had to manage natural and man-made disasters that have had an impact on the mental health of individuals. For example, during 2020, the Philippines was hit by 22 tropical cyclones [18]. Rocha et al. (2021) [18] suggest that has resulted in population displacement, increased socio- economic burden on individuals and families and sudden bereavement which coupled with the pandemic may increase susceptibility to psychological distress.

Islam et al. (2021) [19] considered the psychological impact of multiple "converging systems" (p112) on the healthcare workforce. In a commentary article they argue that Yemeni healthcare workers are faced with longer working hours, no or delayed salary payments, exposure to traumatic events (either from Covid or as a result of war), an increased risk of catching Covid-19 and stigmatization. This, they contend has ed. to increased levels of PTSD, anxiety disorders, lower selfefficacy and "increased detachment from the workplace" (p113).

This has resulted in not only increased demand, but also limited access to mental health services due to mental health staff reallocation to Covid related duties, closure of out-patient services and restriction on businesses providing in person services. The initial United Kingdom Government response to the pandemic necessitated the closure of the majority of publicly funded and all private providers therapy offices requiring therapists to move their practice online.

Online psychotherapy is not new. For example, Christensen et al. [20] reported the effectiveness of the computerized MoodGym CBT programme for anxiety and depression over 15 years ago. More recently, Kuhn et al. [21] explored the effectiveness of a CBT based app designed to reduce PTSD symptoms. They report statistically significant improvements in PTSD symptoms, depressive symptoms and social functioning. NICE produced guidelines for the remote delivery of psychological therapies and recommended a number of 'digital therapies' for various mental health problems including depression, anxiety, substance misuse and body dysmorphic disorder [22]. Interestingly, no digital interventions for trauma specific presentations have been assessed. Additionally, these studies focus on therapy delivered through an app or webpage, where no therapist has direct involvement in the treatment.

Remote delivery of psychotherapy, where the client works with a therapist online or via the telephone [23] has been found to be both effective and acceptable to clients experiencing PTSD symptoms. Knaevelsrud \& Maercker [24] compared CBT delivered via the internet against a waitlist control. They found statistically significant differences in PTSD symptomatology between the two groups after ten sessions delivered over a five-week period. Khun \& Owen [21] carried out a systematic review. They found that PTSD therapy delivered remotely was as effective as in-person treatment although they note that the samples for the papers included in their reviews were primarily military veterans and male, limiting the generalization of their findings.

It is possible that online delivery of therapy could address some of the factors associated with dropout from therapy [25]. Low/ hourly paid work and travel time and cost have previously been identified as factors that drive attrition rates from therapy [26]. The flexibility afforded by online therapy may improve retention rates and, theoretically, clinical outcome [27]. 


\section{Online EMDR}

EMDR therapists have been successfully treating clients remotely over the internet [28]. However, peer reviewed reports on the efficacy of EMDR delivered via the internet are scant. Lenferink Meyerbroker \& Boelen [29] specifically sought papers investigating the efficacy on EMDR online. They found a single uncontrolled study [30] that used internet-based CBT with a web based EMDR tool- iEMDR. The iEMDR is reported to be based on the standard eight-phase protocol outlined by Shapiro [4] adapted for internet delivery. The web link provided in the paper (https://www.rapidtables.com/ tool/EMDR.html) is inactive, thus it is not possible to verify this claim. Fifteen participants started the intervention with 11 completing. Nine participants completed the follow up questionnaires, although the time to follow up is not reported. Spence et al. [30] reported statistically significant improvements in PTSD and anxiety symptoms. The small sample size and lack of control group should counsel against generalizing their findings.

Tarquinio et al. [31] investigated the efficacy of a single EMDR session in reducing anxiety and depression levels as well as the perceived subjective level of disturbance. Seventeen nurses who were in treatment for non-Covid related issues in France had a single session of EMDR, through a video conferencing platform, using the URG-EMDR protocol [24]. They report statistically significant reductions in anxiety, depression, subjective distress, fear of returning to their workplace and fearing for their safety in relation to Covid $(p<0.05)$.

Lazzaroni et al. (2021) [32] used the EMDR protocols for Acute and Recent Traumatic events [33] with a group of adolescents and young adults currently receiving mental healthcare services $(n=50$ (age 1324 years)). The participants received $3 \times 1 \mathrm{~h}$ sessions delivered online. Lazzaroni et al. report significant improvements in anxiety levels and post-traumatic symptomatology $(p=0.05)$ post intervention.

The small sample sizes and lack of control groups in the two studies above should impress the need for caution when interpreting these findings. However, the studies provide some evidence to support the use of EMDR online.

The bilateral stimulation inherent in EMDR therapy can be delivered in several ways from asking clients to follow the therapists' hand or wand as the therapist moves it from side to side [34]. Alternate hand taps and butterfly hugs can also be used, as can smartphones applications that deliver sounds to alternate ears [35].

We are aware of on-going RCT's investigating the effectiveness of EMDR in an online environment [36]. This paper seeks to expand the published literature on using EMDR online.

\section{Aim}

The aim of this service evaluation was to determine evaluate the efficacy of the online delivery of EMDR therapy. Specifically, we sought to answer the following questions;

1. Does EMDR delivered online reduce clinical symptoms of common mental health presentations?

2. Does the level of EMDR accreditation or number of years' experience using EMDR influence the overall client outcome?

\section{Methods}

\section{Recruitment of therapists \& clients}

EMDR therapists were recruited through the EMDR UK and Ireland JISCMail mailing list, and direct email from the EMDR UK Association (circulation 4200 therapists). The JISCMail list is an opt-in discussion forum where subscribers engage in discussion regarding EMDR. It currently has circa 1700 subscribers. Members of both the UK EMDR Association and the JISCMail mailing list are required to have undergone an approved training course and to be practicing under supervision in accordance with EMDR Europe and/or the EMDR International Association standards. We adopted a full population approach to the evaluation and as such all members of either organization were eligible to take part. No exclusion criteria were applied.

A generic mail was sent to all subscribers inviting them to take part. Interested therapists were directed to an online form that detailed the evaluation. Therapists deciding to participate provided details in respect of their level, EMDR experience, and clinical assessment tools used. Participating therapists were then asked to complete an online form giving anonymized information of client's presentation and outcome. As the authors had no direct contact with the clients, therapists were instructed to ensure that clients had given informed consent for their anonymized data to be used. The EMDR UK Association provided support for a random draw of participating therapists where the prize was one of three $£ 20$ gift vouchers. Suggested text regarding consent for therapists to include in their treatment plans/ consent forms was provided. Recruitment of therapists and data collection took place from May 2020-Dec 2020. Google Forms was used to collect data.

\section{Ethics}

Using the algorithm provided by the UK National Health Research Authority and UK Policy Framework for Health and Social Care Research definition of research [37] this project was not classified as research but as a service evaluation [38]. As such formal IRB ethical approval was not required [38]. All participants were 
provided with an electronic information sheet that detailed the background and the aims of the study as well as the requirements of taking part. Respondents gave written informed consent to take part in the evaluation, and all processes undertaken in the evaluation were carried out in accordance with relevant guidelines and policies.

\section{Data collection and analysis}

Author designed surveys were used to collect the data. Data collected from the therapists is reported below and participating therapists then provided anonymized data on their clients including their gender, age, primary psychiatric reason for referral, pre- and post-treatment scores and the outcome measures used in practice. Given the heterogeneity of the assessment and outcomes tools used by EMDR therapists, and this project's status as an evaluation of existing practice we did not put any restriction on the measures used by the therapist. We asked participating therapists to provide overall pre and post treatment scores in relation to the Impact of Events Scale- Revised (IES-R) [39], the General Anxiety Disorder 7 scale (GAD-7) [40], the Public Health Questionnaire 9 scale (PHQ-9) [41] and the PTSD Checklist (PCL-5) [42] and any other assessment scales they normally use for each of their clients.. Thesescales are widely used in PTSD research and clinical practice and have been shown to have good reliability and validity. Twenty five of the 33 therapists used one of the four scales above.

Of the eight that did not use any of these scales, the Subjective Units of Distress and Validity of Cognition scales [4] used routinely in EMDR therapy were reported as outcomes by one of the therapists. Other scales used by therapists were the HADS $(n=1)$ [43], the CORE $(n=1)$ [44], GHQ $(n=1)$ [45], BDI $(n-1)$ [46], BAI $(n=$ 1) [47], AUDIT $(n=1)$ [48], DES-II $(n=1)$ [49], the Worry about Sexual Outcomes scale $(n=1)$ [50], ITQ $(n=2)$ [51], the Moral Disengagement Scale $(n=1)$ [52], the Work and Social Adjustment Scale $(n=2)$ [53], the Driving Cognitions Questionnaire $(n=1)$ [54], and the CRIES $13(n=1)$ [55]. Recognizing the very small numbers using these different assessment tools we have not reported these in this paper.

Descriptive statistics are reported for both therapists and clients. The Shapiro-Wilk test for normality returned non-significant statistics suggesting normal distribution of data. Accordingly, Student t-test was used to identify significant differences between pre- and post-treatment scores. Statistical significance was set at 0.05 throughout. Minimal clinically important differences were calculated using the distribution method [56] where a difference of half the standard deviation is recognised as being clinically important. In order to address the second research question, a third dataset was constructed combining the therapist and client details to allow for cross tabulation of outcome and anonymized therapist details. The Pearson $\mathrm{r}$ correlation statistic was used to explore relationships between the length of time the therapist had been practicing EMDR and each of the clinical outcomes. Analysis of variance (ANOVA) was used to ascertain differences between level of accreditation and clinical outcome. A dichotomous variable of aged 18 or over and under the age of 18 was created. ANOVA was used to explore any differences in outcome by age of the client. JASP software was used to conduct the statistical analysis.

\section{Findings}

Thirty-three therapists provided data on a total of 93 different clients. The therapists' mean length of time since training in EMDR was 8.5 years (sd: 4.8 yrs.: range $(0.5-$ 17.5 yrs). Eight of the therapists were trained to Consultant Level, 10 were accredited EMDR therapists and the 12 had completed initial EMDR training and were working towards accreditation.

Of the 93 clients for whom data was provided, 62 (66\%) identified as female, $30(33 \%)$ as male and 1 as non-binary (1\%). Ages ranged from 10 years to 72 (mean 35.5 sd 15.6 years). Thirteen (14\%) of the clients were under the age of 18 years.

Psychological trauma (simple and complex) was the most common reason for seeking help, followed by anxiety and depression. Table 1 shows the reasons for referral. One of the 93 participants sought help for Covid related problems.

Table 1 Frequencies for 'What was the clients' main presenting problem?'

\begin{tabular}{lll}
\hline & Frequency & Percentage \\
\hline Complex PTSD & 37 & 39.8 \\
Simple PTSD & 19 & 20.4 \\
Anxiety & 14 & 15.1 \\
Depression & 12 & 12.9 \\
OCD & 3 & 3.2 \\
Grief & 2 & 2.2 \\
Health anxiety & 1 & 1.1 \\
"Negative cognitions resulting from & 1 & 1.1 \\
a number of traumatic events led & & \\
to DSH, anxiety and depression" & & 1.1 \\
Phobia & 1 & 1.1 \\
Functional neurological disorder & 1 & 1.1 \\
Wanting to explore negative & 1 & \\
cognitions / "behavioural stuckness" & & 1.1 \\
Psychosis & 1 & \\
\hline
\end{tabular}


Statistically significant and clinically important reductions in the reported client mean scores of the IES(R), the GAD-7, the PHQ-9 and the PCL-5 checklist was found (Table 2). Large effect sizes post-treatment was also found. Minimal clinically important difference thresholds were set at 9.10 (IES(R)), 2.61 (GAD-7), 3.64 (PHQ-9) and 6.64 (PCL-5). No significant relationship was found between length of time trained in EMDR and any of the clinical outcomes, nor was there any significant difference in the association between level of accreditation and clinical outcome. No significant differences were found in any of the four outcomes between genders or between those aged under 18 years and clients aged 18 and over.

\section{Discussion}

This appears to be the first evaluation to report the efficacy of using the standard eight phase EMDR protocol delivered through an online medium. Using real world data, we have shown that EMDR can reduce symptomatology regardless of the age, gender or clinical presentation of the client. The findings also show that length of time practicing EMDR and level of accreditation in EMDR are not associated with outcome, suggesting that EMDR can be used successfully regardless of experience after EMDR training.

Notwithstanding the limitations outlined below, the large effect sizes found are encouraging. A recent systematic review [57] reported a small to moderate pooled effect size (Hedges $g=0.33$ ) [57] across 23 studies using EMDR delivered in the same room as the client for the treatment of PTSD, and a large pooled effect size across 10 studies for the treatment of anxiety disorders (Hedges $\mathrm{g}=1.07)$ [57].

We are also encouraged by the apparent similarities in efficacy reported by therapists working with children and young people and those working with the adult population. This is in keeping with studies that report EMDR as an effective treatment for children and young people (CYP) that have experienced a traumatic event and that clinical improvement using EMDR is independent of demographic variables such as age [58]. For example, in a meta- analysis comparing trauma based approaches to treating PTSD in CYP Khan et al. [58] reported EMDR to be more effective than CBT in reducing PTSD symptoms $(\mathrm{SDM}(95 \% \mathrm{CI})=-0.43(-$ $0.73-0.12), p=0.006)$ and anxiety symptoms (SDM $(95 \% \mathrm{CI})=-0.71(-1.21--0.21), p=0.005)$. Lazzaroni et al. (2021) [32] and Jeon et al. (2017) [59] have shown that age is not correlated with either reductions in PTSD symptoms [32] or Post Traumatic growth [59].

Our findings allude to EMDR being as effective when delivered remotely as face to face, in line with the findings of Kuhn \& Owen [21] We also note that the effect sizes reported here are in excess of those reported in meta-analyses of the CBT interventions $(0.66<\mathrm{g}<0.83)$ delivered via the internet when compared to passive controls (no treatment or wait list control) [60]. Interestingly, they also found that internet CBT was superior to active control groups [60].

As with other psychotherapies, remote delivery of EMDR has significant potential benefits to clients including the reduction in travel time to and from appointments and loss of salary to attend appointments [25] as well as a reduction in stigma associated with mental health treatment [61]. Clients have control over their environment and smartphone apps that deliver clicks via earphones alternately give the client more control of the session [23]. Other applications such as bilateralstimulation.io also provide online platforms for the delivery of the bilateral stimulations used in EMDR. Additionally, internet delivered interventions have been found to be a cost-effective way to deliver psychological interventions [39].

Conversely, when considering working with clients remotely therapists need to be cognisant of potential distractions such as children, deliveries and phone calls during the sessions [23] [27]. Fisher (2021) [23] also acknowledges that the home environment may not always be a safe environment for the client to undergo EMDR therapy and therapists need to be cognisant of this when considering online work with clients.

Having access to the internet is, obviously, a prerequisite for online therapy. Cleofas et al. (2021) [62] surveyed 952 college students and report that computer ownership and access to the internet are associated with lower levels of Covid-19 related anxieties. Rubin (2021) [63] argues that internet access should now be a social determinant of health. She notes that internet access increases with income. Subsequently, therapists need to be

Table 2 Pre-post EMDR scores, ${ }^{a}$ denotes clinically important difference

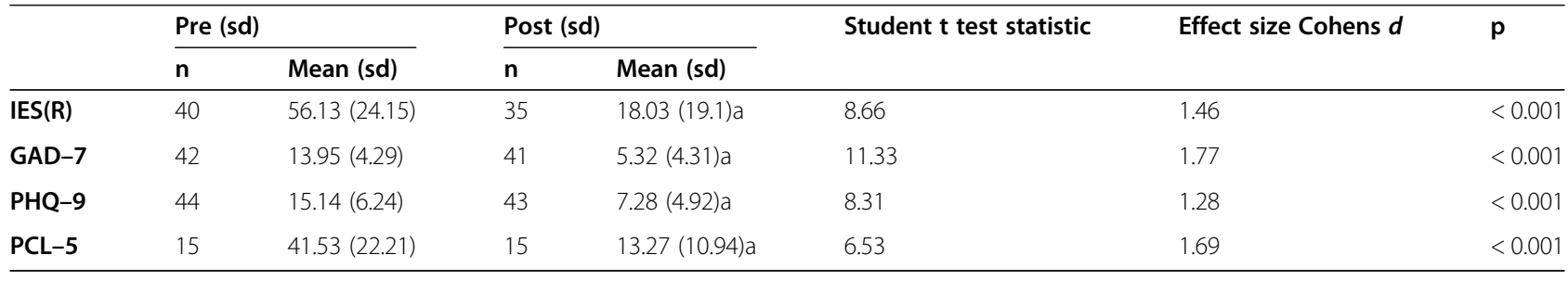


cognisant of the stigma associated with poverty [64] and the potential for those living in poverty to portray a more positive outlook than their current situation [65] when considering moving therapy online.

\section{Limitations}

This evaluation has several limitations. There is a potential for 'gatekeeper bias', where there is the possibility that therapists providing data may only have submitted data for clients that showed improvement. We also recognise that despite high initial interest, a relatively small number of therapists provided client data. This may suggest a self-selecting group with a bias toward online EMDR. The lack of a control group and small sample size of both therapists and clients also precludes us making any generalised claims. Although we note above that client outcome appears to be independent of level of experience as an EMDR therapist, we did not collect data on previous experience of online therapy undertaken by therapist prior to the pandemic. Subsequently, we cannot comment on therapist experience working online and the impact that that may have on clinical outcome. As noted above, we are aware of several on-going Randomised Controlled Trials exploring the effectiveness of EMDR in an online environment that may address these limitations.

\section{Conclusion}

This evaluation appears to be the first paper to report the efficacy of EMDR delivered online using real world practice data. Our findings show that a reduction in clinical symptoms can be achieved using EMDR online, however recognising the limitations of this evaluation we would urge caution in interpreting the findings. Clinical trials examining the clinical and cost effectiveness of online EMDR are required.

The Covid 19 pandemic required EMDR therapists to adopt creative and flexible responses to help meet the needs of the clients [18]. Office closures and travel restrictions to and from work meant that therapists had to move their work to online. The findings of this evaluation suggest that they did so successfully.

\section{Acknowledgements}

Not applicable.

\section{Authors' contributions}

The project was conceived by IM, NF \& JH. All four authors developed that data collection tools. IM and $\mathrm{JH}$ maintained the responses. IM analysed the data and drafted the initial manuscript text. All four authors reviewed and developed the manuscript. The author(s) read and approved the final manuscript.

Authors' information

Not applicable

\section{Funding}

No funding was obtained for the evaluation. EMDR UK sponsored the purchase of $3 \times £ 20$ vouchers for a prize draw for participants.

\section{Availability of data and materials}

The datasets used and/or analysed during the current study available from the corresponding author on reasonable request.

\section{Declarations}

Ethics approval and consent to participate

Using the algorithm provided by the UK National Health Research Authority, and UK Policy Framework for Health and Social Care Research this project was not classified as research but as a service evaluation. As such a service evaluation ethical approval was not required (Health Research Authority 2013). All participants gave informed consent to take part in the evaluation and from the guardians to take part in the evaluation below 16 years old, and all processes undertaken in the evaluation were carried out in accordance with relevant guidelines and policies. A copy of the algorithm was provided to the editors and is available on request.

\section{Consent for publication}

Not Applicable

\section{Competing interests}

The authors declare no competing interests.

\section{Author details}

${ }^{1}$ School of Nursing and Midwifery, Queens University, Belfast, UK. ${ }^{2}$ Clinical Psychologist, Private Therapy Practice, Hove, UK. ${ }^{3}$ Dr. Justin Havens Psychological Therapy, Cheltenham, UK. ${ }^{4}$ Counselling Psychology Solutions, Stratfield Saye, Reading, UK.

Received: 19 March 2021 Accepted: 26 October 2021

Published online: 11 November 2021

\section{References}

1. ISTSS. Adult prevention and early treatment for PTSD guidelines summary [Internet]: ISTSS; 2018. [cited 2021 Mar 18]. Available from: https://istss.org/public-resources/trauma-basics/adult-prevention-and-ea rly-treatment-for-ptsd-

2. Van Ommeren M. Guidelines for the management of conditions specifically related to stress [Internet]. World Health Organisation; 2013. Available from: www.who.int. Accessed 20 Jan 2021.

3. NICE. Post-traumatic stress disorder: NICE Guidelines [NG116] [Internet]. NICE; 2018. [cited 2021 Mar 18]. Available from: https://www.nice.org.uk/ guidance/ng116. Accessed 20 Janu 2021.

4. Shapiro F. Eye Movement Desensitization and Reprocessing (EMDR), 2nd Edition: Basic Principles, Protocols, and Procedures. New York USA: Guilford Press; 2001. p. 959.

5. Proudlock S, Peris J. Using EMDR therapy with patients in an acute mental health crisis. BMC Psychiatry. 2020;20(1):14. https://doi.org/10.1186/s12888019-2426-7.

6. Luo M, Guo L, Yu M, Jiang W, Wang H. The psychological and mental impact of coronavirus disease 2019 (COVID-19) on medical staff and general public - a systematic review and meta-analysis. Psychiatry Res. 2020;291: 113190. https://doi.org/10.1016/j.psychres.2020.113190.

7. Qian Y, Wu K, Xu H, Bao D, Ran F, Wei W, et al. A survey on physical and mental distress among cancer patients during the COVID-19 epidemic in Wuhan, China. J Palliat Med. 2020;23(7):888-9. https://doi.org/10.1089/jpm.2 020.0240

8. Hao F, Tan W, Jiang L, Zhang L, Zhao X, Zou Y, et al. Do psychiatric patients experience more psychiatric symptoms during COVID-19 pandemic and lockdown? A case-control study with service and research implications for immunopsychiatry. Brain Behav Immun. 2020;87:100-6. https://doi.org/10.1 016/j.bbi.2020.04.069.

9. Alkhamees AA, Alrashed SA, Alzunaydi AA, Almohimeed AS, Aljohani MS. The psychological impact of COVID-19 pandemic on the general population of Saudi Arabia. Compr Psychiatry. 2020;102:152192. https://doi.org/10.1016/ j.comppsych.2020.152192. 
10. Farooq SM, Sachwani SAA, Haider SI, Iqbal SA, Parpio YN, Saeed H. Mental health challenges and psycho-social interventions amid COVID-19 pandemic: a call to action for Pakistan. J Coll Physicians Surg--Pak JCPSP. 2020;30(6):59-62. https://doi.org/10.29271/jcpsp.2020. Supp1.S59.

11. Buenaventura RD, Ho JB, Lapid MI. COVID-19 and mental health of older adults in the Philippines: a perspective from a developing country. Int Psychogeriatr. 2020;32(10):1129-33. https://doi.org/10.1017/\$104161022 0000757.

12. Pierce M, Hope H, Ford T, Hatch S, Hotopf M, John A, et al. Mental health before and during the COVID-19 pandemic: a longitudinal probability sample survey of the UK population. Lancet Psychiatry. 2020;7(10):883-92. https://doi.org/10.1016/S2215-0366(20)30308-4

13. González-Sanguino C, Ausín B, Castellanos MÁ, Saiz J, López-Gómez A Ugidos $C$, et al. Mental health consequences during the initial stage of the 2020 coronavirus pandemic (COVID-19) in Spain. Brain Behav Immun. 2020; 87:172-6. https://doi.org/10.1016/i.bbi.2020.05.040.

14. Kaparounaki CK, Patsali ME, Mousa D-PV, Papadopoulou EVK, Papadopoulou KKK, Fountoulakis KN. University students' mental health amidst the COVID19 quarantine in Greece. Psychiatry Res. 2020;290:113111.

15. Wang J, Lloyd-Evans B, Giacco D, Forsyth R, Nebo C, Mann F, et al. Social isolation in mental health: a conceptual and methodological review. Soc Psychiatry Psychiatr Epidemiol. 2017;52(12):1451-61. https://doi.org/10.1007/ s00127-017-1446-1.

16. Huy NT, Duc NTM, Mohammed Alhady ST, Mai LN, Hassan AK, Giang TV, et al. Perceived stress of quarantine and isolation during COVID-19 pandemic: a global survey. Front Psychiatry. 2021;12:656664. https://doi. org/10.3389/fpsyt.2021.656664.

17. TMGH-Global COVID-19 Collaborative. Psychological impacts and posttraumatic stress disorder among people under COVID-19 quarantine and isolation: a global survey. Int J Environ Res Public Health. 2021; 18(11):5719

18. Rocha ICN, dos Santos Costa AC, Islam Z, Jain S, Goyal S, Mohanan P, et al Typhoons during the COVID-19 pandemic in the Philippines: impact of a double crises on mental health. Disaster Med Public Health Prep. 2021;3:1-4. https://doi.org/10.1017/dmp.2021.140.

19. Islam Z, Rocha ICN, Mohanan P, Jain S, Goyal S, dos Santos Costa AC, et al. Mental health impacts of humanitarian crisis on healthcare workers in Yemen. Med Confl Surviv. 2021;37(2):112-7. https://doi.org/10.1080/13623 699.2021.1950519.

20. Christensen H, Griffiths KM, Jorm AF. Delivering interventions for depression by using the internet: randomised controlled trial. BMJ. 2004;328(7434):2650. https://doi.org/10.1136/bmj.37945.566632.EE.

21. Kuhn E, Owen JE. Advances in PTSD treatment delivery: the role of digital technology in PTSD treatment. Curr Treat Options Psychiatry. 2020;7(2):88102. https://doi.org/10.1007/s40501-020-00207-x.

22. NICE. Digital therapies assessed and accepted by the Improving Access to Psychological Therapies Programme (IAPT) | Improving Access to Psychological Therapies (IAPT) [Internet]. NICE. [cited 2021 Oct 6]. Available from: https://www.nice.org.uk/about/what-we-do/our-programmes/nice-a dvice/improving-access-to-psychological-therapies\%2D\%2Diapt-/ submitting-a-product-to-iapt.

23. Fisher N. Using EMDR therapy to treat clients remotely. J EMDR Pract Res. 2021;15(1):73-84. https://doi.org/10.1891/EMDR-D-20-00041.

24. Knaevelsrud C, Maercker A. Internet-based treatment for PTSD reduces distress and facilitates the development of a strong therapeutic alliance: a randomized controlled clinical trial. BMC Psychiatry. 2007;7(1):13. https://doi. org/10.1186/1471-244X-7-13.

25. Acierno R, Knapp R, Tuerk P, Gilmore AK, Lejuez C, Ruggiero K, et al. A noninferiority trial of prolonged exposure for posttraumatic stress disorder: in person versus home-based telehealth. Behav Res Ther. 2017;89:57-65. https://doi.org/10.1016/j.brat.2016.11.009.

26. Barrett B, Byford S, Crawford MJ, Patton R, Drummond C, Henry JA, et al. Cost-effectiveness of screening and referral to an alcohol health worker in alcohol misusing patients attending an accident and emergency department: a decision-making approach. Drug Alcohol Depend. 2006;81(1): 47-54. https://doi.org/10.1016/j.drugalcdep.2005.05.015.

27. Brenes GA, Ingram CW, Danhauer SC. Benefits and challenges of conducting psychotherapy by telephone. Prof Psychol Res Pract. 2011;42(6):543-9. https://doi.org/10.1037/a0026135.
28. Mischler C, Hofmann A, Behnke A, Matits L, Lehnung M, Varadarajan S, Rojas R, Kolassa IT, Tumani $V$. Therapists' Experiences With the Effectiveness and Feasibility of Videoconference-Based Eye Movement Desensitization and Reprocessing. Frontiers in Psychology. 2021;12.

29. Lenferink LIM, Meyerbröker K, Boelen PA. PTSD treatment in times of COVID-19: a systematic review of the effects of online EMDR. Psychiatry Res. 2020;293:113438. https://doi.org/10.1016/j.psychres.2020.113438.

30. Spence J, Titov N, Johnston L, Dear BF, Wootton B, Terides M, et al. Internetdelivered eye movement desensitization and reprocessing (iEMDR): an open trial [Internet]: F1000Research; 2013. [cited 2021 Oct 6]. Available from: https://f1000research.com/articles/2-79

31. Tarquinio C, Brennstuhl M-J, Rydberg JA, Bassan F, Peter L, Tarquinio CL, et al. EMDR in telemental health counseling for healthcare workers caring for COVID-19 patients: a pilot study. Issues Ment Health Nurs. 2021;42(1):314. https://doi.org/10.1080/01612840.2020.1818014.

32. Lazzaroni E, Invernizzi R, Fogliato E, Pagani M, Maslovaric G. Coronavirus disease 2019 emergency and remote eye movement desensitization and reprocessing group therapy with adolescents and young adults: overcoming lockdown with the butterfly hug. Front Psychol. 2021;12: 701381. https://doi.org/10.3389/fpsyg.2021.701381.

33. Shapiro E, Laub B. Early EMDR intervention (EEl): A summary, a theoretical model, and the recent traumatic episode protocol (R-TEP). Journal of EMDR Practice and Research. 2008:2(2):79.

34. Shapiro F, Forrest MS. EMDR: the breakthrough 'eye movement' therapy for overcoming anxiety, stress, and trauma. New York; Oxford: BasicBooks ; Oxford Publicity Partnership; 2004.

35. Marotta-Walters SA, Jain K, DiNardo J, Kaur P, Kaligounder S. A review of mobile applications for facilitating EMDR treatment of complex trauma and its comorbidities. J EMDR Pract Res. 2018;12(1):2-15. https://doi.org/10.1 891/1933-3196.12.1.2.

36. Bates A, Rushbrook S, Shapiro E, Grocott M, Cusack R. CovEMERALD: assessing the feasibility and preliminary effectiveness of remotely delivered eye movement desensitisation and reprocessing following Covid-19 related critical illness: a structured summary of a study protocol for a randomised controlled trial. Trials. 2020;21(1):929. https://doi.org/10.1186/s13063-020-04805-1.

37. NHS Health Research Authority. Defining research [Internet]: NHS Health Research Authority; 2017. [cited 2020 Jan 30]. Available from: www.hradecisiontools.org.uk/research/docs/DefiningResearchTable_Oct2017-1.pdf

38. NHS Health Research Authority. Do I need NHS REC review? [Internet]. NHS Health Research Authority; 2020 [cited 2020 Jan 20]. Available from: http:// www.hra-decisiontools.org.uk/ethics/about.html.

39. Weiss D, Marmar C. The impact of event scale - revised. In: Wilson J, Keane T, editors. Assessing psychological trauma and PTSD. New York: Guildford Press; 1996.

40. Spitzer RL, Kroenke K, Williams JBW, Löwe B. A brief measure for assessing generalized anxiety disorder: the GAD-7. Arch Intern Med. 2006;166(10): 1092-7. https://doi.org/10.1001/archinte.166.10.1092.

41. Kroenke K, Spitzer RL, Williams JB. The PHQ-9: validity of a brief depression severity measure. J Gen Intern Med. 2001;16(9):606-13. https://doi.org/10.1 046/j.1525-1497.2001.016009606.x.

42. Blevins CA, Weathers FW, Davis MT, Witte TK, Domino JL. The posttraumatic stress disorder checklist for DSM-5 (PCL-5): development and initial psychometric evaluation. J Trauma Stress. 2015;28(6):489-98. https://doi. org/10.1002/jts.22059.

43. Stern AF. The hospital anxiety and depression scale. Occup Med. 2014;64(5): 393-4. https://doi.org/10.1093/occmed/kqu024.

44. Barkham M, Mellor-Clark J, Connell J, Evans C, Evans R, Margison F. Clinical Outcomes in Routine Evaluation (CORE) - The CORE measures and system: measuring, monitoring and managing quality evaluation in the psychological therapies. In: Developing and delivering practice-based evidence: a guide for the psychological therapies. Chichester, UK: WileyBlackwell; 2010. p. 175-219. https://doi.org/10.1002/9780470687994.ch8.

45. Schmitz N, Kruse J, Heckrath C, Alberti L, Tress W. Diagnosing mental disorders in primary care: the general health questionnaire $(\mathrm{GHQ})$ and the symptom check list (SCL-90-R) as screening instruments. Soc Psychiatry Psychiat Epidemiol. 1999:34(7):360-6. https://doi.org/10.1007/s001270050156.

46. Beck AT, Beamesderfer A. Assessment of depression: the depression inventory. In: Pichot P, Olivier-Martin R, editors. Modern trends in pharmacopsychiatry [Internet]: S. Karger AG; 1974. p. 151-69. [cited 2021 Oct 7]. Available from: https://www.karger.com/Article/FullText/395074. 
47. Beck AT, Epstein N, Brown G, Steer RA. An inventory for measuring clinical anxiety: psychometric properties. J Consult Clin Psychol. 1988;56(6):893-7. https://doi.org/10.1037/0022-006X.56.6.893.

48. de Meneses-Gaya C, Zuardi AW, Loureiro SR, Crippa JAS. Alcohol Use Disorders Identification Test (AUDIT): an updated systematic review of psychometric properties. Psychol Neurosci. 2009;2(1):83.

49. Carlson EB, Putnam FW. An update on the dissociative experience scale. Dissociation. 1993;6(1):16-27.

50. Sales JM, Spitalnick J, Milhausen RR, Wingood GM, DiClemente RJ, Salazar $L F$, et al. Validation of the worry about sexual outcomes scale for use in STV/ HIV prevention interventions for adolescent females. Health Educ Res. 2009; 24(1):140-52. https://doi.org/10.1093/her/cyn006.

51. Cloitre M, Shevlin M, Brewin CR, Bisson Jl, Roberts NP, Maercker A, et al. The international trauma questionnaire: development of a self-report measure of ICD-11 PTSD and complex PTSD. Acta Psychiatr Scand. 2018;138(6):536-46. https://doi.org/10.1111/acps.12956.

52. Pelton J, Gound M, Forehand R, Brody G. The moral disengagement scale: extension with an American minority sample. J Psychopathol Behav Assess. 2004;26(1):31-9. https://doi.org/10.1023/B:JOBA.0000007454.34707.a5.

53. Mundt JC, Marks IM, Shear MK, Greist JH. The work and social adjustment scale: a simple measure of impairment in functioning. Br J Psychiatry J Ment Sci. 2002;180(5):461-4. https://doi.org/10.1192/bjp.180.5.461.

54. Ehlers A, Taylor JE, Ehring T, Hofmann SG, Deane FP, Roth WT, et al. The driving cognitions questionnaire: development and preliminary psychometric properties. J Anxiety Disord. 2007;21(4):493-509. https://doi. org/10.1016/j.janxdis.2006.08.002.

55. Children and War Foundation. Children's Revised Impact of Event Scale. (CRIES-13). Bergen, Norway: Children and War Foundation; 2005.

56. Rai SK, Yazdany J, Fortin PR, Aviña-Zubieta JA. Approaches for estimating minimal clinically important differences in systemic lupus erythematosus. Arthritis Res Ther. 2015;17(1):143. https://doi.org/10.1186/s13075-015-0658-6.

57. Cuijpers P, van Veen SC, Sijbrandij M, Yoder W, Cristea IA. Eye movement desensitization and reprocessing for mental health problems: a systematic review and meta-analysis. Cogn Behav Ther. 2020;49(3):165-80. https://doi. org/10.1080/16506073.2019.1703801.

58. Khan AM, Dar S, Ahmed R, Bachu R, Adnan M, Kotapati VP. Cognitive behavioral therapy versus eye movement desensitization and reprocessing in patients with post-traumatic stress disorder: systematic review and metaanalysis of randomized clinical trials. Cureus. 2018;10(9):e3250. https://doi. org/10.7759/cureus.3250.

59. Jeon SW, Han C, Choi J, Ko Y-H, Yoon H-K, Kim Y-K. Eye movement desensitization and reprocessing to facilitate posttraumatic growth: a prospective clinical pilot study on ferry disaster survivors. Clin Psychopharmacol Neurosci. 2017;15(4):320-7. https://doi.org/10.9758/cpn.2 017.15.4.320.

60. Kuester A, Niemeyer H, Knaevelsrud C. Internet-based interventions for posttraumatic stress: a meta-analysis of randomized controlled trials. Clin Psychol Rev. 2016;43:1-16. https://doi.org/10.1016/j.cpr.2015.11.004.

61. Levin ME, Krafft J, Levin C. Does self-help increase rates of help seeking for student mental health problems by minimizing stigma as a barrier? J Am Coll Health J ACH. 2018;66(4):302-9. https://doi.org/10.1080/07448481.201 8.1440580 .

62. Cleofas JV, Rocha ICN. Demographic, gadget and internet profiles as determinants of disease and consequence related COVID-19 anxiety among Filipino college students. Educ Inf Technol. 2021. [cited 2021 Sep 27]. Available from: http://link.springer.com/10.1007/s10639-021-10529-9;26(6): 6771-86.

63. Rubin R. Internet access as a social determinant of health. JAMA. 2021, 326(4):298. https://doi.org/10.1001/jama.2021.10972.

64. Bray R, de Laat M, Godinot X, Ugarte A, Walker R. Realising poverty in all its dimensions: a six-country participatory study. World Dev. 2020;134:105025. https://doi.org/10.1016/j.worlddev.2020.105025.

65. Simons AMW, Houkes I, Koster A, Groffen DAl, Bosma H. The silent burden of stigmatisation: a qualitative study among Dutch people with a low socioeconomic position. BMC Public Health. 2018;18(1):443. https://doi.org/1 0.1186/s12889-018-5210-6.

\section{Publisher's Note}

Springer Nature remains neutral with regard to jurisdictional claims in published maps and institutional affiliations.

\section{Ready to submit your research? Choose BMC and benefit from:}

- fast, convenient online submission

- thorough peer review by experienced researchers in your field

- rapid publication on acceptance

- support for research data, including large and complex data types

- gold Open Access which fosters wider collaboration and increased citations

- maximum visibility for your research: over $100 \mathrm{M}$ website views per year

At BMC, research is always in progress.

Learn more biomedcentral.com/submissions 